\title{
Spontaneous emission and quantum discord: comparison of Hilbert-Schmidt and trace distance discord
}

\author{
Lech Jakóbczyk * \\ Institute of Theoretical Physics \\ University of Wroctaw \\ Plac Maxa Borna 9, 50-204 Wroctaw, Poland
}

\begin{abstract}
Hilbert - Schmidt and trace norm geometric quantum discord are compared with regard to their behavior during local time evolution. We consider the system of independent two - level atoms with time evolution given by the dissipative process of spontaneous emission. It is explicitly shown that the Hilbert - Schmidt norm discord has nonphysical properties with respect to such local evolution and cannot serve as a reasonable measure of quantum correlations and the better choice is to use trace norm discord as such a measure.
\end{abstract}

PACS numbers: 03.67.Mn,03.65.Yz,42.50.-p

Keywords: geometric quantum discord, trace norm, Hilbert - Schmidt norm, spontaneous emission

\section{INTRODUCTION}

Characterizing the nature of correlations in composite quantum systems is one of the fundamental problems in quantum theory. When the system is prepared in a pure state, only entanglement is responsible for the presence of quantum correlations. On the other hand, once mixed states are taken into account, the problem becomes much more involved. Some features of separable mixed states are incompatible with a classical description of correlations. The most important among them is that a measurement on a part of composite system in some non-entangled states can induce disturbance on the state of complementary subsystem. Such "non-classical" behavior can be quantified by quantum discord - the most promising measure of bipartite quantum correlations beyond quantum entanglement [1]. For pure states discord coincides with entanglement, but in the case of mixed states discord and entanglement differ significantly. For example it was shown that almost all quantum states have non-vanishing discord [2] and even local operations on the measured part can increase or create quantum discord [3, 4].

In this paper we quantify non-classical correlations which may differ from entanglement by using geometric quantum discord. This quantity is defined in terms of minimal distance of the given state from the set of classically - correlated states, so the proper choice of such a distance is crucial. The measure proposed in [5] uses a Hilbert - Schmidt norm to define a distance in the set of states. This choice has a technical advantage: the minimization process can be realized analytically for arbitrary two-qubit states. Despite of this feature, this measure has some unwanted properties. The most important problem is that it may increase under local operations performed on the unmeasured subsystem [6, 7]. Fortunately, by using other norm in the set of states, this defect can be repaired: the best choice is to use Schatten 1-norm (or trace norm) to define quantum discord [8]. On the other hand, such defined measure is more difficult to compute. The closed for-

\footnotetext{
*ljak@ift.uni.wroc.pl
}

mula for it is known only in the case of Bell - diagonal states or X - shaped two - qubit states [8, 9].

The main scope of this paper is to reconsider the properties of those two measures of quantum discord in a concrete physical system where the quantum channel is given by the time evolution. As a compound system we take two independent two - level atoms not completely isolated from the environment. In this case the time evolution is given by a dissipative process of spontaneous emission. One - sided spontaneous emission in which only one atom emits photons and the other is isolated from the environment, gives the physical realization of local quantum channel. Although it was already established [6, 7], in this framework we can explicitly show that Hilbert - Schmidt norm discord has nonphysical properties with respect to the local evolution and the better choice is to use trace norm. In particular we discuss the local creation of discord when the system is prepared in classical initial state [10-13]. In Ref.[14] we have studied time evolution of Hilbert - Schmidt quantum discord $D_{2}$, now we compare it with the behavior of trace norm quantum discord $D_{1}$. The results of our analysis show that when only the local creation of quantum discord in the classical initial state is considered, $D_{2}$ and $D_{1}$ provide the same information about the evolution of quantum correlations. This is no longer true when the initial states have non - zero discord. Local evolution can increase quantum discord and this phenomenon can be observed by using $D_{1}$ or $D_{2}$. On the other hand, there are initial states with decreasing quantum correlations quantified by $D_{1}$ whereas $D_{2}$ is increasing. The most spectacular manifestation of nonphysical properties of Hilbert - Schmidt norm discord is its behavior during the local evolution of the unmeasured subsystem. $D_{2}$ not only increases for a large class of initial discordant states (at the same time $D_{1}$ obviously decreases) but also it can increase even when the local evolution of the measured subsystem leads to decreasing $D_{2}$. This shows again that in contrast to trace norm discord, Hilbert - Schmidt norm discord cannot serve as a reasonable measure of quantum correlations. 


\section{GEOMETRIC MEASURES OF QUANTUM DISCORD}

We start with the introduction of the standard notion of geometric quantum discord [5]. When a $d \otimes d$ bipartite system $A B$ is prepared in a state $\rho$ and we perform local measurement on the subsystem $A$, almost all states $\rho$ will be disturbed due to such measurement. The (one-sided) geometric discord $D_{2}(\rho)$ can be defined as the minimal disturbance, measured by the squared Hilbert-Schmidt distance, induced by any projective measurement $\mathbb{P}^{A}$ on subsystem $A$ i.e.

$$
D_{2}(\rho)=\frac{d}{d-1} \min _{\mathbb{P}^{A}}\left\|\rho-\mathbb{P}^{A}(\rho)\right\|_{2}^{2},
$$

where

$$
\|a\|_{2}=\sqrt{\operatorname{tr} a a^{*}}
$$

Here we adopt normalized version of the geometric discord, introduced in Ref. [15]. In the case of two qubits, there is an explicit expression for $D_{2}$ [5]:

$$
D_{2}(\rho)=\frac{1}{2}\left(\|\boldsymbol{x}\|^{2}+\|T\|_{2}^{2}-k_{\max }\right),
$$

where the components of the vector $\boldsymbol{x} \in \mathbb{R}^{3}$ are given by

$$
x_{k}=\operatorname{tr}\left(\rho \sigma_{k} \otimes \mathbb{1}\right),
$$

the matrix $T$ has elements

$$
T_{j k}=\operatorname{tr}\left(\rho \sigma_{j} \otimes \sigma_{k}\right)
$$

and $k_{\max }$ is the largest eigenvalue of the matrix $\boldsymbol{x} \boldsymbol{x}^{T}+T T^{T}$. Despite of being easy to compute, the measure $D_{2}$ fails as a quantifier of quantum correlations, since it may increase under local operations on the unmeasured subsystem [6]. In the present paper we explicitly show that one-sided spontaneous emission of the unmeasured atom can create additional discord quantified by $D_{2}$ in the system of two independent atoms. Such defect of $D_{2}$ originates in the properties of Hilbert Schmidt norm, which manifests also in the case of entanglement [16].

To repair this defect, one considers other norms in the set of quantum states. The best choice is to use the trace norm (or Schatten 1-norm) and define [8]

$$
D_{1}(\rho)=\min _{\mathbb{P}^{A}}\left\|\rho-\mathbb{P}^{A}(\rho)\right\|_{1},
$$

where

$$
\|a\|_{1}=\operatorname{tr}|a|
$$

$D_{1}$ has desired properties with respect to the local operations on unmeasured subsystem, but its computation is much more involved. Analytic expression for $D_{1}$ is known only for limited classes of two - qubits states, including Bell - diagonal [8] and $X$ - shaped mixed states [9]. In the present paper, we consider $X$ - shaped two - qubit states

$$
\rho=\left(\begin{array}{cccc}
\rho_{11} & 0 & 0 & \rho_{14} \\
0 & \rho_{22} & \rho_{23} & 0 \\
0 & \rho_{32} & \rho_{33} & 0 \\
\rho_{41} & 0 & 0 & \rho_{44}
\end{array}\right),
$$

where all matrix elements are real and non - negative. The quantity $D_{1}$ for such states can be computed as follows. Let $x=2\left(\rho_{11}+\rho_{22}\right)-1$ and

$\alpha_{1}=2\left(\rho_{23}+\rho_{14}\right), \quad \alpha_{2}=2\left(\rho_{23}-\rho_{14}\right), \quad \alpha_{3}=1-2\left(\rho_{22}+\rho_{33}\right)$.

Then [9]

$$
D_{1}(\rho)=\sqrt{\frac{a \alpha_{1}^{2}-b \alpha_{2}^{2}}{a-b+\alpha_{1}^{2}-\alpha_{2}^{2}}},
$$

where

$$
a=\max \left(\alpha_{3}^{2}, \alpha_{2}^{2}+x^{2}\right), \quad b=\min \left(\alpha_{3}^{2}, \alpha_{1}^{2}\right) .
$$

Notice that we use normalized version of $D_{1}$ and the formula (II.10) is not valid in the case when $x=0$ and

$$
\left|\alpha_{1}\right|=\left|\alpha_{2}\right|=\left|\alpha_{3}\right|
$$

In such a case, one can use general prescription how to compute $D_{1}$, also given in Ref. [9] (eq. (65)).

In the case of pure states, $D_{1}$ as well as $D_{2}$ give the same information about quantum correlations as entanglement measured by negativity

$$
N(\rho)=\left\|\rho^{\mathrm{PT}}\right\|_{1}-1,
$$

where $\rho^{\mathrm{PT}}$ denotes partial transposition of $\rho$. In the case of mixed states, entanglement and discord significantly differ. For example for two - qubit Bell - diagonal states one finds that [8]

$$
D_{1} \geq \sqrt{D_{2}} \geq N
$$

The inequality $\sqrt{D_{2}} \geq N$ was proved to be valid for all two - qubit mixed states [15], and it is conjectured that (I.14) is also valid for all two - qubit states.

To show that inequalities in (II.14) can be sharp, consider the following family of states [17]

$$
\rho_{\theta}=\left(\begin{array}{cccc}
\frac{1}{2} \cos ^{2} \theta & 0 & 0 & \frac{1}{4} \sin 2 \theta \\
0 & 0 & 0 & 0 \\
0 & 0 & \frac{1}{2} & 0 \\
\frac{1}{4} \sin 2 \theta & 0 & 0 & \frac{1}{2} \sin ^{2} \theta
\end{array}\right)
$$

where $\theta \in[0, \pi / 2]$. By direct computation, one can check that

$$
N\left(\rho_{\theta}\right)=\frac{\sqrt{6-2 \cos 4 \theta}-2}{4},
$$

whereas

$$
D_{2}\left(\rho_{\theta}\right)=\min \left(\frac{1}{2} \sin ^{2} \theta, \frac{1}{4} \sin ^{2} 2 \theta\right)
$$

and

$$
D_{1}\left(\rho_{\theta}\right)=\frac{1}{2} \sin 2 \theta
$$


Notice that

$$
D_{1}\left(\rho_{\theta}\right)>\sqrt{D_{2}\left(\rho_{\theta}\right)}>N\left(\rho_{\theta}\right.
$$

if $\theta \in(0, \pi / 4)$ and

$$
D_{1}\left(\rho_{\theta}\right)=\sqrt{D_{2}\left(\rho_{\theta}\right)}>N\left(\rho_{\theta}\right)
$$

for $\theta \in[\pi / 4, \pi / 2]$ (see FIG.1).

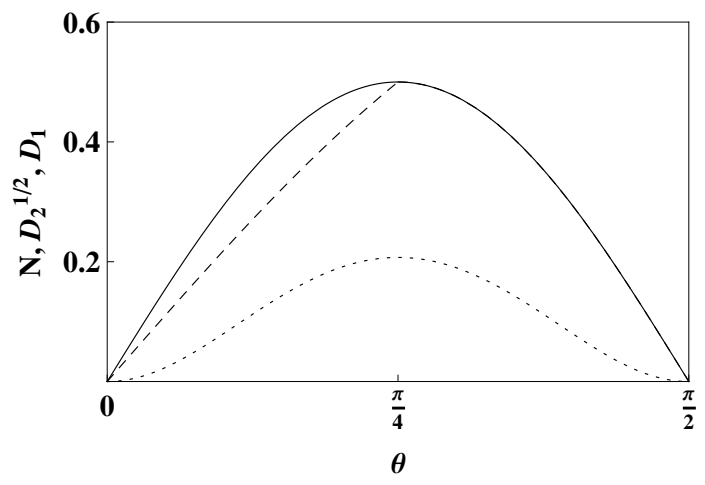

FIG. 1: $N$ (doted line), $\sqrt{D_{2}}$ (dashed line) and $D_{1}$ (solid line) as a functions of the parameter $\theta$ for the states (II.15).

\section{LOCAL DYNAMICS INDUCED BY SPONTANEOUS EMISSION AND GEOMETRIC DISCORD}

\section{A. One - sided spontaneous emission}

Consider a system of two independent two - level atoms (atom $A$ and atom $B$ ) interacting with environment at zero temperature. In this study we take into account only the dissipative process of spontaneous emission, so the dynamics of the system is given by the master equation [18]

$$
\frac{d \rho}{d t}=L_{A B} \rho, \quad L_{A B}=L_{A}+L_{B},
$$

where for $k=A, B$

$$
L_{k}=\frac{\gamma_{0}}{2}\left(2 \sigma_{-}^{k} \rho \sigma_{+}^{k}-\sigma_{+}^{k} \sigma_{-}^{k} \rho-\rho \sigma_{+}^{k} \sigma_{-}^{k}\right) .
$$

In the above equation $\sigma_{ \pm}^{A}=\sigma_{ \pm} \otimes \mathbb{1}, \sigma_{ \pm}^{B}=\mathbb{1} \otimes \sigma_{ \pm}$and $\gamma_{0}$ is the single atom spontaneous emission rate. Local evolution of the atom $A$ is given by "one-sided" spontaneous emission generated only by the generator $L_{A}$ i.e.

$$
\rho_{t, A}=T_{t}^{A} \rho, \quad T_{t}^{A}=e^{t L_{A}} .
$$

In this case the atom $A$ spontaneously emits photons, whereas the atom $B$ is isolated from the environment. Similarly we can consider one-sided spontaneous emission of the atom $B$ i.e. the evolution

$$
\rho_{t, B}=T_{t}^{B} \rho, \quad T_{t}^{B}=e^{t L_{B}} .
$$

In what follows we consider the $X$ - shaped initial states (II.8), where the matrix elements of $\rho$ are given with respect to the basis $|e\rangle_{A} \otimes|e\rangle_{B},|e\rangle_{A} \otimes|g\rangle_{B},|g\rangle_{A} \otimes|e\rangle_{B},|g\rangle_{A} \otimes|g\rangle_{B}$ and $|g\rangle_{k},|e\rangle_{k}, k=A, B$ are the ground states and excited states of atoms $A$ and $B$. For such initial state, the state $\rho_{t, A}$ has the following matrix elements

$$
\begin{aligned}
& \left(\rho_{t, A}\right)_{11}=e^{-\gamma_{0} t} \rho_{11}, \\
& \left(\rho_{t, A}\right)_{22}=e^{-\gamma_{0} t} \rho_{22}, \\
& \left(\rho_{t, A}\right)_{33}=\left(1-e^{-\gamma_{0} t}\right) \rho_{11}+\rho_{33}, \\
& \left(\rho_{t, A}\right)_{44}=\left(1-e^{-\gamma_{0} t}\right) \rho_{22}+\rho_{33}, \\
& \left(\rho_{t, A}\right)_{14}=e^{-\gamma_{0} t / 2} \rho_{14}, \\
& \left(\rho_{t, A}\right)_{23}=e^{-\gamma_{0} t / 2} \rho_{23} .
\end{aligned}
$$

Similarly

$$
\begin{aligned}
& \left(\rho_{t, B}\right)_{11}=e^{-\gamma_{0} t} \rho_{11}, \\
& \left(\rho_{t, A}\right)_{22}=\left(1-e^{-\gamma_{0} t}\right) \rho_{11}+\rho_{22}, \\
& \left(\rho_{t, A}\right)_{33}=e^{-\gamma_{0} t} \rho_{33}, \\
& \left(\rho_{t, A}\right)_{44}=\left(1-e^{-\gamma_{0} t}\right) \rho_{33}+\rho_{44}, \\
& \left(\rho_{t, A}\right)_{14}=e^{-\gamma_{0} t / 2} \rho_{14}, \\
& \left(\rho_{t, A}\right)_{23}=e^{-\gamma_{0} t / 2} \rho_{23} .
\end{aligned}
$$

Notice that in contrast to the usual process of spontaneous emission, for the the one-sided emissions, there are non-trivial asymptotic states: one can check that for any initial state $\rho$ when $t \rightarrow \infty$

$$
\rho_{t, A} \rightarrow P_{g}^{A} \otimes \operatorname{tr}_{A} \rho
$$

and

$$
\rho_{t, B} \rightarrow \operatorname{tr}_{B} \rho \otimes P_{g}^{B}
$$

where $P_{g}^{A}, P_{g}^{B}$ are projections on the ground states of the atom $A$ and $B$ respectively.

\section{B. Time evolution of $D_{1}$ and $D_{2}$}

Now we study quantum correlations in the states $\rho_{t, A}$ and $\rho_{t, B}$ defined above. We start with trace distance geometric discord. In the state $\rho_{t, A}$ we have

$$
D_{1}\left(\rho_{t, A}\right)=\sqrt{\frac{a(t) \alpha_{1}(t)^{2}-b(t) \alpha_{2}(t)^{2}}{a(t)-b(t)+\alpha_{1}(t)^{2}-\alpha_{2}(t)^{2}}},
$$

where

$$
\begin{aligned}
& \alpha_{1}(t)=2\left(\rho_{14}+\rho_{23}\right) e^{-\gamma_{0} t / 2}, \\
& \alpha_{2}(t)=2\left(\rho_{23}-\rho_{14}\right) e^{-\gamma_{0} t / 2} \\
& \alpha_{3}(t)=2\left(\rho_{11}-\rho_{22}\right) e^{-\gamma_{0} t}-2\left(\rho_{11}+\rho_{33}\right)+1, \\
& x(t)=2\left(\rho_{11}+\rho_{22}\right) e^{-\gamma_{0} t}-1
\end{aligned}
$$


and

$$
\begin{aligned}
& a(t)=\max \left(\alpha_{3}(t)^{2}, \alpha_{2}(t)^{2}+x(t)^{2}\right), \\
& b(t)=\min \left(\alpha_{3}(t)^{2}, \alpha_{1}(t)^{2}\right) .
\end{aligned}
$$

Similarly

$$
D_{1}\left(\rho_{t, B}\right)=\sqrt{\frac{\widetilde{a}(t) \widetilde{\alpha}_{1}(t)^{2}-\widetilde{b}(t) \widetilde{\alpha}_{2}(t)^{2}}{\widetilde{a}(t)-\widetilde{b}(t)+\widetilde{\alpha}_{1}(t)^{2}-\widetilde{\alpha}_{2}(t)^{2}}},
$$

where

$$
\begin{aligned}
& \widetilde{\alpha}_{1}(t)=\alpha_{1}(t), \quad \widetilde{\alpha}_{2}(t)=\alpha_{2}(t), \quad \widetilde{x}(t)=x, \\
& \widetilde{\alpha}_{3}(t)=2\left(\rho_{11}-\rho_{33}\right) e^{-\gamma_{0} t}-2\left(\rho_{11}+\rho_{22}\right)+1
\end{aligned}
$$

and

$$
\begin{aligned}
& \widetilde{a}(t)=\max \left(\widetilde{\alpha}_{3}(t)^{2}, \widetilde{\alpha}_{2}(t)^{2}+\widetilde{x}(t)^{2}\right), \\
& \widetilde{b}(t)=\min \left(\widetilde{\alpha}_{3}(t)^{2}, \widetilde{\alpha}_{1}(t)^{2}\right) .
\end{aligned}
$$

Concerning $D_{2}$, one finds

$$
D_{2}\left(\rho_{t, A}\right)=\min \left(f_{1}(t), f_{2}(t), f_{3}(t)\right)
$$

where

$$
\begin{aligned}
f_{1}(t)= & 4\left(\rho_{14}^{2}+\rho_{23}^{2}\right) e^{-\gamma_{0} t}, \\
f_{2}(t)= & 4\left(\rho_{11}^{2}+\rho_{22}^{2}\right) e^{-2 \gamma_{0} t}+2\left[\left(\rho_{14}-\rho_{23}\right)^{2}\right. \\
& \left.-2 \rho_{11}\left(\rho_{11}+\rho_{33}\right)-2 \rho_{22}\left(\rho_{22}+\rho_{44}\right)\right] e^{-\gamma_{0} t} \\
& +\left(\rho_{11}+\rho_{33}\right)^{2}+\left(\rho_{22}+\rho_{44}\right)^{2}, \\
f_{3}(t)= & 4\left(\rho_{11}^{2}+\rho_{22}^{2}\right) e^{-2 \gamma_{0} t}+2\left[\left(\rho_{14}+\rho_{23}\right)^{2}\right. \\
& \left.-2 \rho_{11}\left(\rho_{11}+\rho_{33}\right)-2 \rho_{22}\left(\rho_{22}+\rho_{44}\right)\right] e^{-\gamma_{0} t} \\
& +\left(\rho_{11}+\rho_{33}\right)^{2}+\left(\rho_{22}+\rho_{44}\right)^{2} .
\end{aligned}
$$

Similarly

$$
D_{2}\left(\rho_{t, B}\right)=\min \left(\widetilde{f}_{1}(t), \widetilde{f}_{2}(t), \widetilde{f}_{3}(t)\right)
$$

where

$$
\begin{aligned}
\widetilde{f}_{1}(t)= & f_{1}(t), \\
\widetilde{f}_{2}(t)= & 2\left(\rho_{11}-\rho_{33}\right)^{2} e^{-2 \gamma_{0} t}+2\left[\left(\rho_{14}-\rho_{23}\right)^{2}\right. \\
& \left.-\left(\rho_{11}-\rho_{33}\right)\left(\rho_{11}+\rho_{22}-\rho_{33}-\rho_{44}\right)\right] e^{-\gamma_{0} t} \\
& +\left(\rho_{11}+\rho_{22}\right)^{2}+\left(\rho_{33}+\rho_{44}\right)^{2} \\
& -2\left(\rho_{11}+\rho_{22}\right)\left(\rho_{33}+\rho_{44}\right), \\
\widetilde{f}_{3}(t)= & 2\left(\rho_{11}-\rho_{33}\right)^{2} e^{-2 \gamma_{0} t}+2\left[\left(\rho_{14}+\rho_{23}\right)^{2}\right. \\
& \left.-\left(\rho_{11}-\rho_{33}\right)\left(\rho_{11}+\rho_{22}-\rho_{33}-\rho_{44}\right)\right] e^{-\gamma_{0} t} \\
& +\left(\rho_{11}+\rho_{22}\right)^{2}+\left(\rho_{33}+\rho_{44}\right)^{2} \\
& -2\left(\rho_{11}+\rho_{22}\right)\left(\rho_{33}+\rho_{44}\right) .
\end{aligned}
$$

\section{Classically correlated initial states}

We choose as initial states the following $X$-shaped states

$$
\rho_{c}=\left(\begin{array}{cccc}
w & 0 & 0 & s \\
0 & \frac{1}{2}-w & s & 0 \\
0 & s & w & 0 \\
s & 0 & 0 & \frac{1}{2}-w
\end{array}\right)
$$

where

$$
0<w<\frac{1}{2}, \quad 0<s \leq s_{\max }
$$

and

$$
s_{\max }=\sqrt{\frac{1}{2} w-w^{2}} .
$$

One can check that

$$
D_{1}\left(\rho_{c}\right)=D_{2}\left(\rho_{c}\right)=0
$$

so $\rho_{c}$ are only classically correlated. Notice that for such initial states

$$
\begin{aligned}
& \alpha_{1}(t)=4 s e^{-\gamma_{0} t / 2}, \quad \alpha_{2}(t)=0 \\
& \alpha_{3}(t)=(1-4 w)\left(1-e^{-\gamma_{0} t}\right), \quad x(t)=e^{-\gamma_{0} t}-1,
\end{aligned}
$$

so

$$
a(t)=\max \left(\alpha_{3}(t)^{2}, x(t)^{2}\right)=\alpha_{3}(t)^{2}
$$

and

$$
D_{1}\left(\rho_{t, A}\right)=\frac{4 s\left(1-e^{-\gamma_{0} t}\right)}{G(t)}
$$

where

$$
G(t)=\sqrt{16 s^{2}+g(t)-\min \left(16 s^{2}, g(t)(1-4 w)^{2}\right)}
$$

and

$$
g(t)=2\left(\cosh \gamma_{0} t-1\right) .
$$

One can check that (III.25) as a function of $t$ grows from zero to some maximal value and then asymptotically vanishes. So for any initial state (III.19) there is a local generation of transient quantum correlations measured by $D_{1}$. The most efficient production of discord is when $w=1 / 4$ and in that case, the maximum is achieved for $s=1 / 4$ i.e. for initial state of the form

$$
\rho_{0}=\frac{1}{2}|+\rangle\langle+|\otimes|+\rangle\left\langle+\left|+\frac{1}{2}\right|-\right\rangle\langle-|\otimes|-\rangle\langle-|,
$$

where

$$
| \pm\rangle=\frac{1}{\sqrt{2}}(|e\rangle \pm|g\rangle)
$$

Due to the properties of trace distance, $D_{1}$ is non- increasing under general local operations on subsystem $B$, so it is equal to 
zero for all $t$. In our model of local evolutions we can check it explicitly: for initial states (III.19) $\widetilde{\alpha}_{1}(t)=4 s e^{-\gamma_{0} t / 2}$ but $\widetilde{\alpha}_{2}(t)=\widetilde{\alpha}_{3}(t)=\widetilde{x}(t)=0$, so $D_{1}\left(\rho_{t, B}\right)=0$.

Now we consider the same problem, but using Hilbert Schmidt distance discord $D_{2}$. In the case of initial state $\rho_{c}$ we have

$$
\begin{aligned}
& f_{1}(t)=8 s^{2} e^{-\gamma_{0} t} \\
& f_{2}(t)=\left(1-4 w+8 w^{2}\right)\left(1-e^{-\gamma_{0} t}\right)^{2}, \\
& f_{3}(t)=\left(1-4 w+8 w^{2}\right)\left(1-e^{-\gamma_{0} t}\right)^{2}+8 s^{2} e^{-\gamma_{0} t} .
\end{aligned}
$$

Notice that $f_{3}(t)>f_{2}(t)$ and $f_{1}(t)$ is decreasing from the value $8 s^{2}$ to zero, whereas $f_{2}(t)$ is increasing from zero to the value $1-4 w+8 w^{2}$, as $t$ goes to infinity. So there is the time $t_{\max }$ at which those functions are equal and $D_{2}$ defined by the formula (III.15) grows from zero to some maximal value and then asymptotically vanishes. Similarly as in the case of $D_{1}$, maximal production of discord is for the initial state (III.28). Moreover, since $\widetilde{f}_{2}(t)=0, D_{2}\left(\rho_{t, B}\right)=0$. Thus we have shown that as far as the process of local generation of quantum correlations out of classical correlations is concerned, $D_{1}$ and $D_{2}$ give the similar information (see FIG.2).

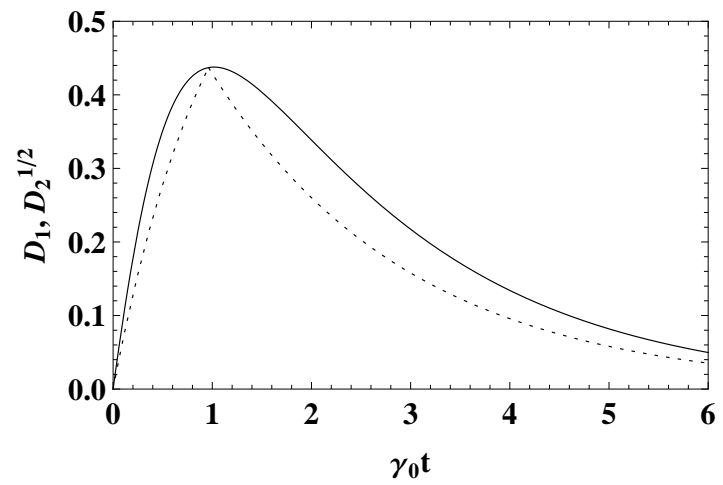

FIG. 2: Time evolution of $D_{1}$ (solid line) and $\sqrt{D_{2}}$ (dotted line) for the initial state $\rho_{0}$, under one - sided emission of the atom $A$.

\section{Some initial states with non - zero discord}

Local evolution can also increase the initial non - zero discord. To show this in our model, consider the states of the form

$$
\rho_{d}=\left(\begin{array}{cccc}
w & 0 & 0 & s \\
0 & w & s & 0 \\
0 & s & \frac{1}{2}-w & 0 \\
s & 0 & 0 & \frac{1}{2}-w
\end{array}\right)
$$

where as in the case of $\rho_{c}$

$$
0<w<\frac{1}{2} \quad \text { and } \quad 0<s \leq s_{\max }
$$

In contrast to the states (III.19), the states $\rho_{d}$ have non - zero discord, for all $w \in(0,1 / 2)$ (except of $w=1 / 4)$ and admissible $s$. One can check that

$$
D_{1}\left(\rho_{d}\right)=\frac{4 s|1-4 w|}{\sqrt{16 s^{2}+(1-4 w)^{2}}}
$$

and

$$
D_{2}\left(\rho_{d}\right)=\min \left(8 s^{2}, 2\left(2 w-\frac{1}{2}\right)^{2}\right) .
$$

We start the analysis of time evolution of quantum correlations by considering first Hilbert - Schmidt discord $D_{2}$. For the local spontaneous emission of the atom $A$ and initial state $\rho_{d}$ we have

$$
\begin{aligned}
& f_{1}(t)=8 s^{2} e^{-\gamma_{0} t} \\
& f_{2}(t)=\frac{1}{2}-4 w e^{-\gamma_{0} t}+8 w^{2} e^{-2 \gamma_{0} t} \\
& f_{3}(t)=\frac{1}{2}-4 w e^{-\gamma_{0} t}+8 w^{2} e^{-2 \gamma_{0} t}+8 s^{2} e^{-\gamma_{0} t}
\end{aligned}
$$

Since $f_{3}(t)>f_{2}(t)$, only the relations between $f_{1}(t)$ and $f_{2}(t)$ are crucial for the behavior of $D_{2}$. Notice that $f_{1}(t)$ decreases form the value $8 s^{2}$ and goes to zero. On the other hand, the function $f_{2}(t)$ may be increasing or decreasing, depending on the value of the parameter $w$. It can be shown that if $0<$ $w \leq 1 / 4, f_{2}(t)$ increases, whereas if $1 / 4<w<1 / 2, f_{2}(t)$ initially decreases and then start to increase. The production of an additional discord can happen only when $f_{2}$ is increasing function of $t$ and when $f_{1}(0)>f_{2}(0)$ i.e. when $0<w<1 / 4$ and

$$
8 s^{2}>\frac{1}{2}-4 w+8 w^{2}
$$

The condition (III.36) gives some restrictions on the possible values of the parameter $w$ in order to obtain production of discord. If we put $s_{\max }$ into the inequality (III.36), we obtain the critical value of $w$ given by

$$
w_{c}=\frac{1}{8}(2-\sqrt{2}) .
$$

One can show that if $0<w \leq w_{c}$, each pair $(w, s)$ where $0<$ $s \leq s_{\max }$ defines the initial state with only decreasing $D_{2}$. On the other hand, if $w_{c}<w<1 / 4$, a pair $\left(w, s_{\max }\right)$, gives the initial state with increasing discord. The values of $w$ greater then $1 / 4$ always give decreasing $D_{2}$.

Now we consider trace norm discord $D_{1}$. For the initial state $\rho_{d}$ we obtain

$$
\begin{aligned}
& \alpha_{1}(t)=4 s e^{-\gamma_{0} t / 2}, \quad \alpha_{2}(t)=\alpha_{3}(t)=0, \\
& x(t)=4 w e^{-\gamma_{0} t}-1,
\end{aligned}
$$

so

$$
D_{1}\left(\rho_{t, A}\right)=\frac{4 s\left|1-4 w e^{-\gamma_{0} t}\right|}{\sqrt{16 s^{2}+e^{\gamma_{0} t}\left(1-4 w e^{-\gamma_{0} t}\right)^{2}}} .
$$




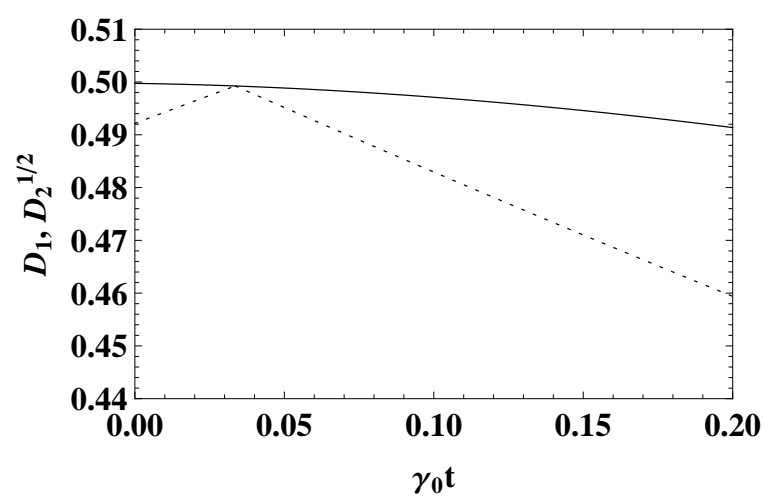

FIG. 3: Time evolution of $D_{1}$ (solid line) and $\sqrt{D_{2}}$ (dotted line) for the initial state $\rho_{d}$ with $w=0.076, s=0.179$.

Since the numerator of the right hand side of (III.39) increases only when $0<w \leq 1 / 4$ and denominator always increases, for such values of $w, D_{1}$ may increase for some period of time. Detailed analysis of the formula (III.39) shows that similarly as in the case of $D_{2}$, there is the critical value $\bar{w}_{c}$ such that for any pair $(w, s), 0<w \leq \bar{w}_{c}$ and admissible $s$, the corresponding initial state gives decreasing discord $D_{1}$, whereas any pair $\left(w, s_{\max }\right), \bar{w}_{c}<w<1 / 4$, defines initial state with growing $D_{1}$. The crucial for this analysis is the fact that $\bar{w}_{c}$ is slightly larger then $w_{c}\left(\bar{w}_{c} \approx 0.0777\right.$ and $\left.w_{c} \approx 0.0732\right)$, so there are the initial states corresponding to $w \in\left(w_{c}, \bar{w}_{c}\right)$ such that two measures of geometric discord behave very differently: $D_{2}$ grows for some period of time, whereas $D_{1}$ decreases for all $t$ (see FIG.3). For the initial states with values of $w$ between $\bar{w}_{c}$ and $1 / 4$, two measures of discord behave similarly: for a finite period of time, $D_{1}$ and $D_{2}$ grow to some maximal value and then start to decrease (FIG.4). Notice also that for the initial states with $w>1 / 4$ the function (III.39) decreases to zero at finite time $t_{0}=(\ln 4 w) / \gamma_{0}$ and then starts to grow to some maximal value. The similar behavior can be observed also in the case of $D_{2}$ (FIG.5).

Now we consider time evolution given by local spontaneous

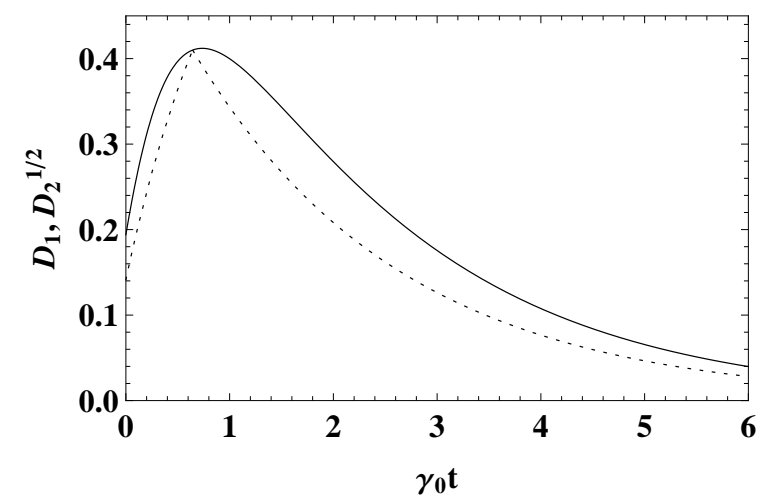

FIG. 4: Time evolution of $D_{1}$ (solid line) and $\sqrt{D_{2}}$ (dotted line) for the initial state $\rho_{d}$ with $w=0.2, s=0.2$.

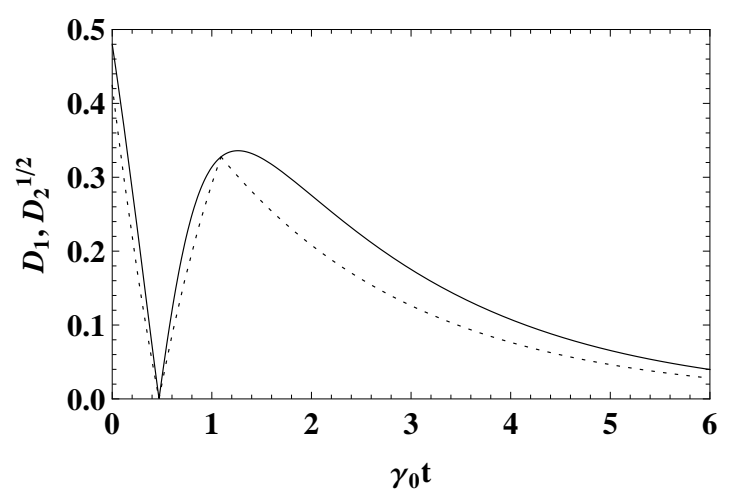

FIG. 5: Time evolution of $D_{1}$ (solid line) and $\sqrt{D_{2}}$ (dotted line) for the initial state $\rho_{d}$ with $w=0.4, s=0.2$.

emission of atom $B$. Since $D_{1}$ is non - increasing under local operations on subsystem $B$, we only consider the properties of $D_{2}\left(\rho_{t, B}\right)$. It is given by formula (III.17), where

$$
\begin{aligned}
& \widetilde{f}_{1}(t)=8 s^{2} e^{-\gamma_{0} t} \\
& \widetilde{f}_{2}(t)=c-c e^{-\gamma_{0} t}+\frac{1}{2} c e^{-2 \gamma_{0} t}, \\
& \widetilde{f}_{3}(t)=c-c e^{-\gamma_{0} t}+\frac{1}{2} c e^{-2 \gamma_{0} t}+8 s^{2} e^{-\gamma_{0} t}
\end{aligned}
$$

and

$$
c=1-8 w+16 w^{2} .
$$

One can check that in this case the function $\widetilde{f}_{2}(t)$ is increasing for all values of $w$ (except of $w=1 / 4$ ) and the condition that $\widetilde{f}_{1}(0)>\widetilde{f}_{2}(0)$ is the same as in the case of evolution of atom $A$. Thus for all pairs $\left(w, s_{\max }\right)$ where $w \in\left(w_{c}, 1 / 2\right], w \neq 1 / 4$, the corresponding initial state gives increasing $D_{2}$ under the local evolution of unmeasured subsystem $B$. This happens even in the case when local evolution of measured atom $A$ leads to

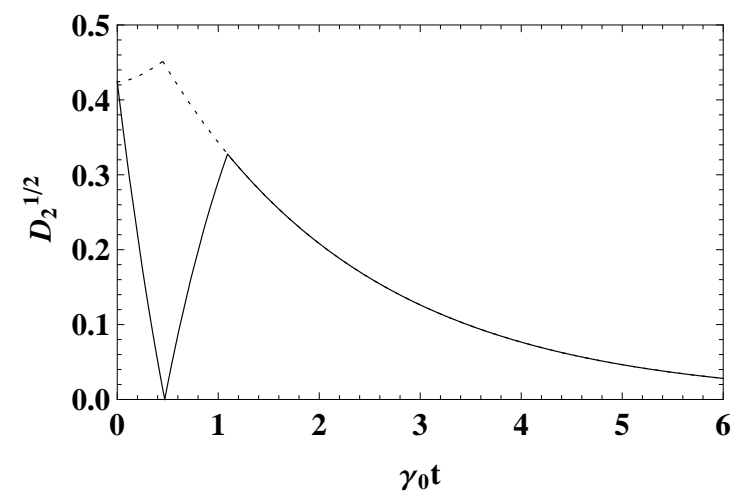

FIG. 6: Time evolution of $\sqrt{D_{2}}$ given by local emission of atom $A$ (solid line) versus local emission of atom $B$ (dotted line), for the initial state $\rho_{d}$ with $w=0.4, s=0.2$. 
decreasing $D_{2}$. It explicitly shows nonphysical properties of Hilbert - Schmidt discord $D_{2}$ (FIG.6).

\section{E. Conclusions}

We have studied local time evolution of quantum correlations given by geometric quantum discord in the system of independent two - level atoms interacting with environment at zero temperature. The dynamics is induced by the process of spontaneous emission and we have local dynamics when only one atom emits and the other is isolated from the environment. Within this model we have compared the properties of Hilbert - Schmidt distance discord $D_{2}$ and trace distance discord $D_{1}$. When the only local generation of discord in the classically correlated initial states is considered, $D_{1}$ and $D_{2}$ provide the similar information: for a large class of initial states with zero discord and local evolution of measured subsystem, $D_{1}$ as well as $D_{2}$ grows from zero to some maximal value and then decay to zero. Moreover, local evolution of unmeasured subsystem in both cases gives the same result: $D_{1}$ and $D_{2}$ are equal to zero. Local quantum operations can also increase the existing discord. We have shown that this phenomenon occurs also in our model of local evolutions for a large class of initially discordant states. In contrast to the previous case, now the behavior of $D_{1}$ and $D_{2}$ significantly differ. First of all $D_{2}$ increases under the local evolution of unmeasured subsystem. It happens even in such cases when it decreases under the evolution of a measured subsystem, which is manifestly nonphysical. Moreover, $D_{2}$ can increase during the evolution of a measured subsystem whereas at the same time $D_{1}$ decreases. All those properties of $D_{2}$ suggests that it is not a reasonable measure of quantum correlations - more promising is to use trace distance discord $D_{1}$.
[1] H. Ollivier and W.H. Zurek, Phys. Rev. Lett. 88, 017901(2001)

[2] A. Ferraro et al. Phys. Rev. A 81, 052318(2010)

[3] A. Streltsov, H. Kampermann and D. Bruss, Phys. Rev. Lett. 107, 170502(2011)

[4] X. Hu et al. Phys. Rev. A 84, 022113(2011)

[5] B. Dakić, V. Vedral and C. Brukner, Phys. Rev. Lett. 105, 190502(2010)

[6] M. Piani, Phys. Rev. A 86, 034101(2012)

[7] T. Tufarelli et al. Phys. Rev. A 86, 052326(2012)

[8] F.M. Paula, Thiago R. de Oliveira and M.S. Sarandy, Phys. Rev. A 87, 064101(2013)

[9] F. Ciacarello, T. Tufarelli and V. Giovannetti, New J. Phys. 16, 013038(2014).

[10] F. Ciccarello and V. Giovannetti, Phys. Rev. A 85, 010102(2012)
[11] F. Ciccarello and V. Giovannetti, Phys. Rev. A 85, 022108(2012)

[12] M. Gessner, E.-M. Laine, H.-P. Breuer and J. Piilo, Phys. Rev. A 85, 052122(2012)

[13] S. Campbell et al. Phys. Rev. A 84, 052316(2011)

[14] M. Gwóźdź and L.Jakóbczyk, Quantum Inf. Process. 13, 171(2014).

[15] D. Girolami and G. Adesso, Phys. Rev. A 84, 052110(2011)

[16] M. Ozawa, Phys. Lett. A 268, 158(2000).

[17] H. Mehri-Dehnavi, B. Mizra, H. Mohammadzadeh and R. Rahimi, Ann. Phys. 326, 1320(2011)

[18] G.S. Agarwal, Quantum Statistical Theories of Spontaneous Emission and their Relation to Other Approaches,Springer, Berlin, 1974. 